\title{
Neuro-Modulation in Dorsal Premotor Cortex Facilitates Human Multi-Task Ability
}

\author{
Yusuke Suzuki $^{1,2}$, Eiichi Naito ${ }^{1,3,4}$ \\ ${ }^{1}$ Cognitive Mechanisms Laboratories, Advanced Telecommunications Research Institute International, Kyoto, Japan \\ ${ }^{2}$ Graduate School of Information Science, Nara Institute of Science and Technology, Nara, Japan \\ ${ }^{3}$ CiNet, National Institute of Information and Communications Technology, Kyoto, Japan \\ ${ }^{4}$ Graduate School of Medicine, Osaka University, Osaka, Japan \\ Email: eiichi.naito@nict.go.jp
}

Received March 7, 2012; revised April 3, 2012; accepted April 10, 2012

\begin{abstract}
Humans are limited in their ability to perform multiple cognitive-motor tasks in parallel. In eight participants, we examined whether transcranial direct current stimulation (tDCS) to dorsal premotor cortex (PMD) could attenuate a delay of reaction time (RT) while the participants responded to two visual stimuli presented in temporally close succession. We provided anodal, cathodal, or sham tDCS while the participants performed a task requiring two choice responses or a control task requiring two fixed responses. When the interval between the two stimuli was shorter, the RTs were delayed in both tasks, but anodal tDCS shortened them only in the former task, probably by promoting the response selection function of PMD. Non-invasive neuro-modulation to the brain can boost human ability to multi-task.
\end{abstract}

Keywords: Transcranial Direct Current Stimulation (tDCS); Psychological Refractory Period (PRP); Dorsal Premotor Cortex (PMD); Response Selection

\section{Introduction}

Humans are limited in their ability to perform multi-task. When people are presented with two discrete stimuli (S1 and S2) in temporally close succession, and required a fast discrete response for each stimulus, the response to S2 is usually delayed compared with the case where the same stimulus is presented alone [1-4]. This behavioral phenomenon is called a psychological refractory period (PRP [1-4]). The PRP is a representative behavioral phenomenon, which shows computational limitation in human brain processing when people perform multiple cognitive-motor tasks in parallel.

Since early findings, it has been generally accepted that temporal overlapping between the processes for the first reaction (R1) and the second reaction (R2) generates the PRP effect $[1,2,4,5]$. Thus, when a time lag between S1 and S2 (stimulus onset asynchrony; SOA) is smaller, a robust PRP effect is observed [1,2,4,5]. In addition to the effect of the SOA length, the process that selects an appropriate response from multiple choices (= response selection) in each reaction may also augment the PRP effect $[1,2,4-6]$. Indeed, when a response selection is required in each reaction $[7,8]$, the PRP effect can be also observed in the R1 in addition to the R2.

A recent human neuroimaging study has revealed the neural correlates of the PRP effect. When people per- formed a PRP task requiring a response selection, greater activity in the dorsal premotor cortex (PMD) was observed in association with the greater PRP effect [9]. This is most probably because the PMD is a key locus for response selection in the primate's brain [10-13], and multiple choices in reaction might overload the neuronal processes in the PMD beyond its normal computational limitation. If this view is correct, we may expect that the PRP effect particularly observed under the requirement of response selection in reaction can be attenuated by facilitating neuronal processing in the PMD.

To test this hypothesis, we took an advantage of transcranial direct current stimulation (tDCS). This non-invasive stimulation can modulate neuronal processing in a stimulated cerebral region in a polarity-specific manner [14-18]. For example, anodal tDCS to the primary motor cortex (M1) is capable of increasing motor cortical excitability by upregulating the membrane potentials of M1 cells, while cathodal tDCS decreases it [14]. Thus, if we extend this principle to other cerebral regions, we may assume that anodal tDCS increases the likelihood that the cells will fire in a stimulated cerebral region, while cathodal tDCS will generate the opposite effects. Based on this assumption, we expected that anodal tDCS to the PMD could promote the neural processing in the PMD, so that the tDCS may facilitate its response selection pro- 
cess and attenuate the PRP effect.

In the present study, we prepared two PRP tasks; one task required a choice response (response selection) in each reaction (choice-choice task; CC task), and the other required a simple fixed response in each reaction (simple-simple task; SS task). First, we evaluated the degree of PRP effect in the two tasks to confirm a greater PRP effect in the CC task. Second, in the tDCS experiments, we examined whether the PRP effect likely observed in the CC task could be attenuated when we provided anodal tDCS to the PMD while participants performed this task. Through these experiments, we explored the possibility that this non-invasive neuro-modulation technique could boost multi-tasking ability in healthy people.

\section{Materials and Methods}

\subsection{Participants}

Eight right-handed, healthy volunteers (seven male and one female; aged 23 - 42) participated both in the evaluation of PRP effect and in the tDCS experiments. We examined their handedness using the Oldfield questionnaire [19]. The laterality quotient was 100 in all participants. The Ethical Committee of the National Institute of Communication and Technology (NICT) approved the study, and all participants provided written informed consent. The experiment was carried out according to the princeples and guidelines of the Declaration of Helsinki (1975).

\subsection{General Task Procedure}

The participants were seated in front of a computer monitor (17-inch 1704FP, Dell Inc., Texas, USA; black panel in Figure 1) located $50 \mathrm{~cm}$ in front of them. A trial started with the presentation of an exclamation mark (!) in the center of the monitor. After this stimulus, various visual stimuli (both distracter and target stimuli) were presented on both right and left sides of a fixation point $(+)$ displayed in the center of the monitor. Through a trial, they were encouraged to gaze at this point to restrict free eye movements (Figure 1). The distracter stimuli were six different symbols often used in a slot machine (Figure 1). Each distracter stimulus was randomly presented for $150 \mathrm{~ms}$ with an inter-stimulus-interval (ISI) of 150 ms. In the series of distracter presentation, a target stimulus (either a green 7 or red 7) randomly appeared. The participants were instructed that the stimulus presented on the left side of the fixation point was S1, and that on the right side was S2. S1 always preceded S2. The participants had to first react with their right hand to S1 (R1) and then to S2 (R2) as quickly as possible in the following two PRP tasks. One trial was completed when they responded to S2. The inter-trial interval was $6 \mathrm{~s}$. Regarding SOA between S1 and S2, four different conditions
(50, 150, 500, or $1000 \mathrm{~ms})$ were prepared in each PRP task, and these were randomized across trials in an experimental block of each task.

In the CC (choice-choice) task, they were asked to make a choice response both in the first and in the second reactions. They had to press the $\mathrm{N}$ key on a keyboard with their index finger when the red 7 was presented, whereas they had to press the $\mathrm{M}$ key with their middle finger in the case of green 7 (Figure 1). The same stimulus-response rule was applied for both R1 and R2. Thus, in a trial of this task, response selection was required in each reaction.

In the SS (simple-simple) task, the participants were required to make a simple fixed response to each of S1 and S2; simply pressing the $\mathrm{N}$ key with their index finger whenever either the green or red 7 was presented as S1 (R1). Likewise, they simply pressed the M key with their middle finger whenever either stimulus was presented as S2 (R2). Thus, in this task, no response selection was required for each reaction. The total number of key-press on each of $\mathrm{M}$ and $\mathrm{N}$ key was balanced between the CC and SS tasks. We adopted this stimulus-response rule, because in the pilot experiment we observed a similar PRP effect when we asked the participants to switch the rule, i.e. $\mathrm{M}$ key (middle finger) response to $\mathrm{S} 1$ and $\mathrm{N}$ key (index finger) response to $\mathrm{S} 2$.

In both CC and SS tasks, we measured reaction time (RT) for each R1 and R2, i.e. RT1 and RT2 respectively. Each RT was defined as the time from the onset of the target stimulus to the reaction (key-press). Since we presented the same visual stimuli to trigger the finger responses in both PRP tasks, possible RT differences between tasks should be attributed to the differences in the type of reaction assigned to each task, rather than to general effects such as attention to visual stimuli.

\subsection{Evaluation of PRP Effect}

First, we evaluated the degree of PRP effect in each of the CC and SS tasks. The participants performed these tasks on separate days, and thus they completed two experiments. The orders of the CC and SS tasks were randomized across participants. In each experiment, the participants completed two blocks, with a 30-min break between them. Each block contained 100 trials and lasted for $20 \mathrm{~min}$. Before starting each experiment, they practiced 100 trials of the corresponding task.

\section{4. tDCS Experiments}

As expected, in the evaluation of PRP effect, we found greater PRP effect in the CC task (see Results). Thus, we focused on the effect of tDCS on the CC task. The same participants performed the same CC task three times while receiving different types of tDCS (Table 1). 


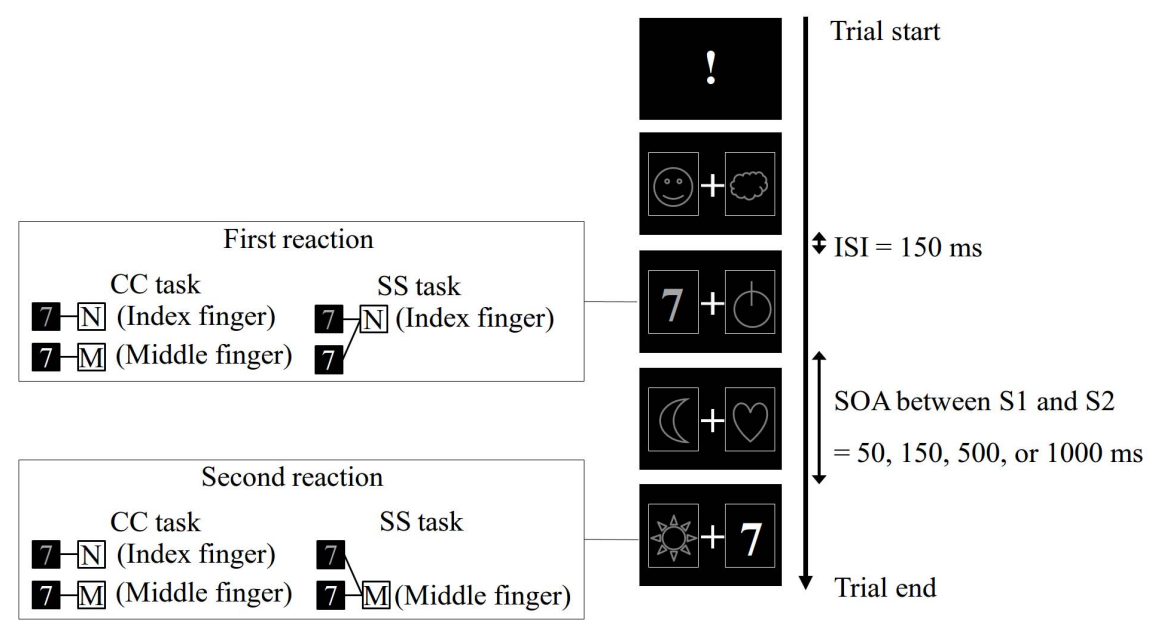

Figure 1. Example of trial of PRP tasks. For details, please see the text. ISI, inter-stimulus-interval; SOA, stimulus onset asynchrony; CC task, choice-choice task; SS task, simple-simple task.

tDCS was applied to the left dorsal premotor cortex (PMD). Current was applied through a saline-soaked pair of surface sponge electrodes $\left(35 \mathrm{~cm}^{2}\right)$, delivered by a battery-driven stimulator (DC-stimulator-Pulse M, neuroConn, Germany) with an output of $1.5 \mathrm{~mA}$. Fade-in and -out times were 5 seconds. The target electrode (either anodal or cathodal) was placed over the left PMD according to the previous tDCS [17] and TMS studies $[20,21]$. We defined the PMD as the cortex $2.5 \mathrm{~cm}$ anterior to the "hotspot" of the left primary motor cortex (M1), which was identified by a single-pulse transcranial magnetic stimulation (TMS) (Magstim 200, Magstim Company Ltd., Wales, UK) based on the international 10 - 20 system. The target electrode was placed over the PMD site in a way that covered its anterior part. Thus, the electrode likely stimulated the PMD and its anterior cortex mainly. The reference electrode was affixed contra-laterally above the orbita.

As in the evaluation of PRP effect, each experiment consisted of two blocks (Blocks 1 and 2), with a break (30 min) between them. Each block contained 100 trials and lasted for 20 min. In each experiment, sham tDCS was always given in Block 1, and either real anodal, real cathodal, or sham tDCS was delivered in Block 2 (Table 1). Eventually, sham-anodal, sham-cathodal, and sham-sham experiments were performed and each was conducted in a separate day. The order of these experiments was pseudo-randomized across participants without informing them.

The sham $\mathrm{tDCS}$ was a short-period stimulation using either anodal or cathodal tDCS. Use of either stimulation was pseudo-randomized between and within the participants. We started delivering either stimulation just before the task was initiated. However, the stimulation lasted for only $1 \mathrm{~min}$ and was terminated without informing the participants during the task. We used this stimulation as
Table 1. tDCS experimental design.

\begin{tabular}{lll}
\hline Tasks & Block 1 & Block 2 \\
\hline CC & Sham & Sham \\
CC & Sham & Cathodal \\
CC & Sham & Anodal \\
SS & Sham & Anodal \\
\hline
\end{tabular}

sham stimulation because tingling/itchy sensation accompanied with tDCS was normally faded out within 1 min regardless of its types [22] so that the participants can not notice the termination when the stimulation was terminated after $1 \mathrm{~min}$. This type of experimental maneuver is often used to control placebo effects from tDCS [16, 23,24].

Real anodal tDCS in the sham-anodal experiment and real cathodal tDCS in the sham-cathodal experiment was applied for $20 \mathrm{~min}$ respectively; the stimulation started just before the task was initiated in Block 2 and lasted until the end of the block. We additionally prepared a sham-sham experiment in which we delivered sham tDCS in both blocks. This experiment was included to simply evaluate possible effects of task repetition and/or practice when the participants performed the block of CC tasks twice without receiving any substantial effects from tDCS.

To examine the net effect of real anodal or cathodal tDCS, we thought the use of ineffective sham stimulation was essential. Because real tDCS likely includes placebo effects, i.e. participants may expect some effects from the stimulation. To genuinely evaluate the net effect, direct comparison between RT obtained under the influence of real tDCS and that obtained under the influence of sham tDCS within an experiment would be the most appropri- 
ate way. Furthermore, the order of sham and real tDCS in an experiment was also important because behavioral improvement under the influence of sustained tDCS remains even after the cessation of tDCS [25]. Thus, if one wants to evaluate the real tDCS effect by comparing with its control (= sham) within an experiment, a sham tDCS block, where no substantial effects are expected, must come first before a real tDCS block. By these reasons, we prepared three experiments consisting of sham-sham, shamcathodal, and sham-anodal for the CC task.

Finally, since we found that the anodal tDCS to the PMD shortened the RTs in the CC task (see Results), we checked if this shortening was exclusively observed in the CC task. To this end, we conducted an extra experiment in which we provided anodal tDCS while the participants performed the SS task. This was done after the three experiments for the CC task were completed, and the same sham-anodal type of experiment was conducted (Table 1). Thus, this experiment was performed as a control for the anodal tDCS effect in the CC task.

After the participants completed all four tDCS experiments (Table 1), we verbally asked the following two questions to each participant. These questions were: 1) "In the series of experiments, we used two different types of stimuli. Did you notice the difference?" and 2) "During the task, we sometimes terminated the stimulation. Were you aware of this?” All participants reported that they could not distinguish the two types of stimuli and that they were not aware of the stimulus termination. Thus, the present sham stimulation seemed to be an appropriate control, and RT change under the influence of real tDCS compared with that under the influence of sham tDCS can be attributed to the net effect of real tDCS.

\subsection{Data Analysis}

In the analysis for the tDCS experiments, we used the data from the last 70 trials in each block by excluding the first 30 trials. This was done because certain duration of tDCS seems to be necessary to invoke real tDCS effects [14], so that we could expect that the tDCS effects become robust in the trials after certain period of tDCS. Indeed, when we checked the tDCS effects in the first 30 trials (during 5 min after the initiation of tDCS), no significant tDCS effects emerged (see Results). The analysis using the last 70 trials was also done in the evaluation of PRP effect.

In the evaluation of PRP effect, we first calculated the mean for RT1 and RT2 in each SOA condition of the CC and SS tasks for each participant. In this analysis, we excluded trials where the participants made incorrect responses and also trials with correct responses, which exceeded two standard deviations of the mean of the raw data. Eventually, the individual mean for each SOA condition was calculated from 16 trials on average. Then, we calculated the grand mean across participants. This was conducted for RT1 (Figure 2(a)) and RT2 (Figure 2(b)), separately. We also calculated the mean RT difference between RT1 and RT2 in each SOA condition of the CC and SS tasks in each participant, and the grand mean across participants was also calculated (Figure 2(c)). For statistical evaluation, we performed two-factorial [task (CC or SS; 2) $\times \operatorname{SOA}(50,150,500$, and $1000 \mathrm{~ms} ; 4)]$ analysis of variance (ANOVA; repeated measurement; $\mathrm{n}=$ 8) for the RT and the RT difference. We used statistical software (PASW Statistics ver. 17.0, SPSS Inc., Illinois, USA). The alpha level was set at 0.05 . We reported $\eta_{p}^{2}$ as the estimate of the effect size.

In the evaluation of PRP effect, we observed a robust PRP effect under the 50-ms condition, and this disappeared whenever SOA was longer than $500 \mathrm{~ms}$ (Figure 2(c); c.f. [26,27]). Thus, in the tDCS analysis, we focused on the data obtained from the $50 \mathrm{~ms}$ and $500 \mathrm{~ms}$ conditions. First, we calculated the mean RT in each

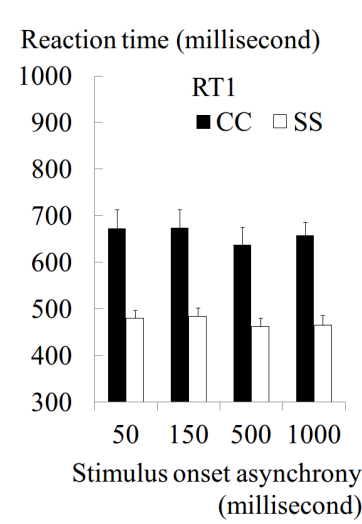

(a)

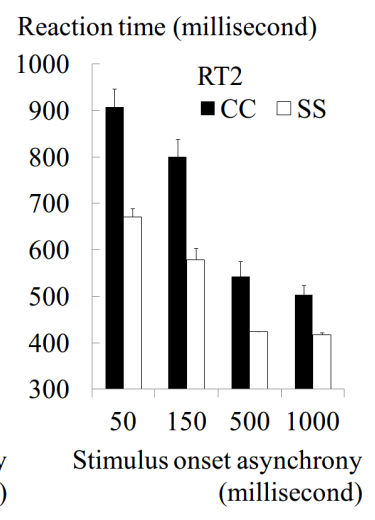

(b)

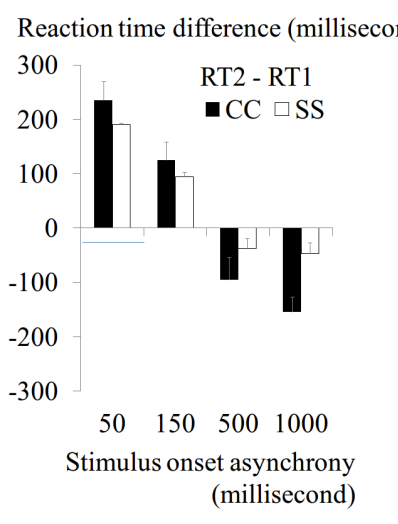

(c)

Figure 2. Results from evaluation of PRP effect. Mean RT (a: RT1 and b: RT2) and mean RT difference between RT1 and RT2 (c) across participants are shown for each SOA condition of CC (black bars) and SS (white bars) tasks. Error bars indicate standard errors of means across participants. The Y-axis indicates RT (ms). 
block of all tDCS conditions for each participant. The 50-ms and 500-ms conditions were separately treated. The individual mean for each condition was calculated from 16 trials on average. These were conducted for the CC and SS tasks respectively. Next, we computed the RT difference between Block 1 and Block 2 for each participant. The difference in RT1 and RT2 was separately computed. Finally, the grand mean across participants was calculated (Figure 3). To confirm whether the RT shortening in the $50 \mathrm{~ms}$ condition of the CC task under the influence of anodal tDCS was significant when compared with the baseline $(=0 \mathrm{~ms})$, one-sample t-test was conducted. We also performed the two-factorial [tDCS type (sham, anodal, or cathodal; 3$) \times$ reaction $(\mathrm{RT} 1$ or RT2; 2)] ANOVA for the RT difference in the CC task.

\section{Results}

\subsection{Evaluation of PRP Effect}

Since the participants practiced the tasks before the experiments, all of them successfully performed both the CC and SS tasks at a high accuracy rate (over $90 \%$ on average).

RT1 in the CC task became significantly longer than that in the SS task in all SOA conditions [main effect of task: $\mathrm{F}(1,7)=59.2, \mathrm{p}<0.001, \eta_{p}^{2}=0.89$, Figure 2(a)]. We also found that RT1 under the 50-ms and 150-ms conditions became longer than that under the 500 -ms and 1000-ms conditions. This was commonly observed in the CC and SS tasks [main effect of SOA: F(3, 21) $=6.3, \mathrm{p}=$ $0.003, \eta_{p}^{2}=0.47$, Figure 2(a)], though RT1 delay under the 50-ms condition compared with that under the 500-ms condition was longer in the former task (35-ms and 19-ms in the CC and SS tasks, respectively).

RT2 in the CC task was also longer than that in the SS

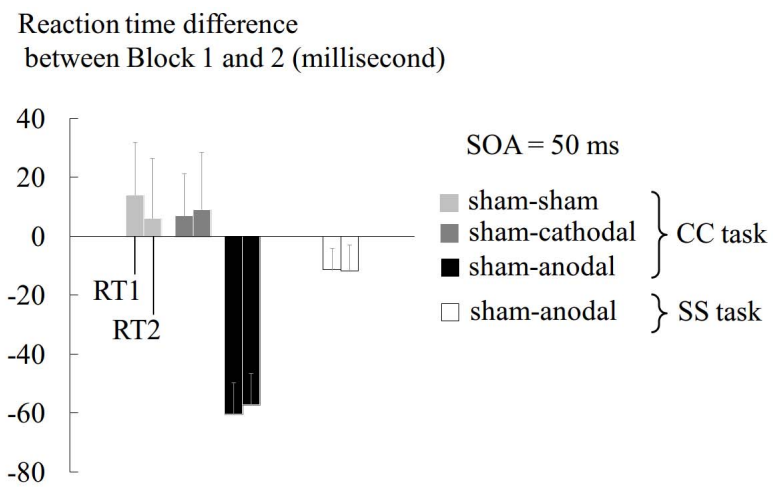

(a) task in all SOA conditions (Figure 2(b)). In both tasks, unlike RT1, RT2 under the 50-ms and 150-ms conditions became much longer than that under the 500-ms and 1000-ms conditions, which was actually shorter than the corresponding RT1 (Figures 2(a) and (b)). Despite no dif- ference in RT1 between the 50-ms and 150-ms condi- tions (Figure 2(a)), RT2 in the 50-ms condition became longer than that in the 150-ms condition in both tasks (Figure 2(b)). This represented the PRP effect normally reported in previous studies (= effect of SOA length; see Introduction).

When we computed the RT difference between RT1 and RT2 (Figure 2(c)), we found that the difference largely depended on the length of SOA. The difference (both degrees of prolongation and shortening) was consistently greater in the CC task than in the SS task in all SOA conditions. The two-way ANOVA for the RT difference revealed a significant interaction between these factors $\left[\mathrm{F}(3,21)=15.4, \mathrm{p}<0.001, \eta_{p}^{2}=0.69\right]$, meaning that the influence of SOA length on RT2 was marked in the CC task.

\section{2. tDCS Experiments}

Among the three (sham-sham, sham-anodal, and shamcathodal) experiments prepared for the CC task, we found RT shortening only in Block 2 of the sham-anodal experiment (Figure 3). This RT shortening was consistently observed in all participants. When we analyzed the RT difference between the blocks in this experiment, both RT1 and RT2 in the 50-ms condition became shorter by $60 \mathrm{~ms}$ on average in Block 2 (Figure 3(a)). The shortening of RT1 and RT2 was significant when compared with the baseline (one-sample t-test; $\mathrm{df}=7, \mathrm{p}<$ 0.001 and $\mathrm{p}<0.002$, respectively). Furthermore, the

Reaction time difference

between Block 1 and 2 (millisecond)

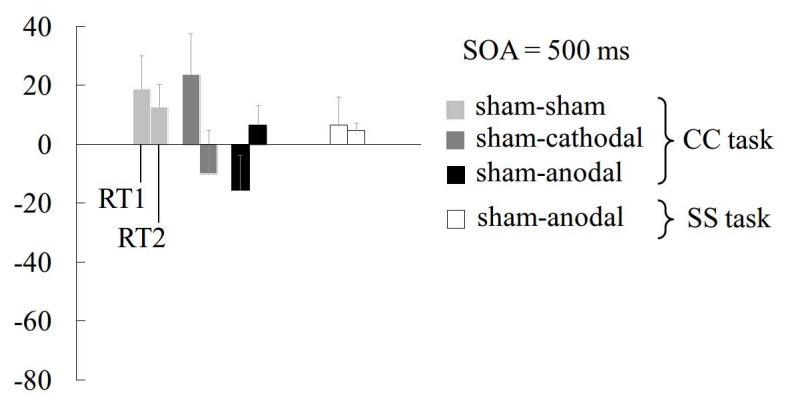

(b)

Figure 3. Results from tDCS experiments. Mean RT difference between Block 1 and Block 2 across participants are shown for RT1 and RT2 in 50-ms (a) and 500-ms (b) conditions of CC and SS tasks. In both panels, the light grey bars indicate the data under sham tDCS, the grey bars indicate the data under cathodal tDCS, and the black bars indicate the data under anodal tDCS in the CC task. The white bars indicate data under anodal tDCS in the SS task. The left bar in each pair represents the RT1 data and the right bar represents the RT2 data. The error bars indicate standard errors of means across participants. The Y-axis indicates the difference in RT (ms). 
two-way ANOVA revealed a significant main effect of the tDCS type on the RT difference between the blocks $\left[\mathrm{F}(2,14)=3.94, \mathrm{p}<0.05, \eta_{p}^{2}=0.36\right]$. Thus, the RT shortening was associated with application of anodal tDCS and could not be attributed to simple effects of task repetition and/or practice, which should have been observed in the sham-sham experiment. When we performed the same analysis using only the first 30 trials, the main effect of tDCS did not reach the significance level ( $p=0.17$ ). Thus, the tDCS effect did not seem to be robust in the first 30 trials.

Another important finding was that even in the shamanodal experiment, RT shortening was only observed under the 50-ms condition, but not under the 500-ms condition (Figures 3(a) and (b)). Thus, the anodal tDCS short- ened the RTs specifically under the experimental con- dition in which a robust PRP effect was observed, and this finding refuted the view that the tDCS simply pro- moted neuronal processes to shorten the RTs in general. Finally, even under the 50-ms condition, anodal tDCS did not significantly shorten the RTs in the SS task (Fig- ure 3(a)).

Viewed collectively, anodal tDCS shortened the RTs under the 50-ms condition where the PRP effect emerged, specifically in the CC tasks in which response selection was required in each consecutive reaction.

\section{Discussion}

In the evaluation of PRP effect, we found that both RT1 and RT2 were significantly longer in the CC task than in the SS task in all SOA conditions (Figures 2(a) and (b)). This means that the response selection required in the CC task needed longer processing time than that for fixed response in the SS task. In addition, both RT1 and RT2 were longer under the 50-ms condition than under the 500-ms condition in both CC and SS tasks (Figures 2(a) and (b)). The RT1 was obtained from the first reaction (R1), which was not preceded by any other reaction processes. But under the 50-ms condition, the processes for the R1 and R2 should have overlapped substantially. In this sense, unlike the conventional PRP effect (= marked delay in the RT2) under the 50-ms condition (Figure 2(c)), the RT1 prolongation should also be considered to be a PRP effect as partially discussed in previous studies $[7,8]$. Finally, the RT difference between RT1 and RT2 under the 50-ms condition was greater in the CC task than in the SS task (Figure 2(c)). Thus, in the evaluation of PRP effect, we confirmed that the PRP effect becomes robust when multiple response selection processes are temporally overlapping, as was typically observed in the 50-ms condition of the CC task.

Anodal tDCS was capable of attenuating the PRP effects (Figure 3(a)). It is generally accepted that anodal
tDCS increases the likelihood that neurons will fire, thereby lowering their threshold to generate action potentials [14]. Under these neuronal states, the neurons will fire in a quite efficient way when inputs reach them [14]. This may lead to facilitation of local neural processing in a stimulated neuronal circuit. Thus, the present anodal tDCS likely facilitated the neural processing of the stimulated cortex, i.e. PMD.

Even though cathodal tDCS might have had potential to generate some effects, this had no impact on RTs in the present study (Figure 3). This reason remains unclear. However, the finding indicates that the cathodal neuromodulation effect is not a simple reversal of the anodal one, as reported in previous studies [28-30]. Indeed, a recent study has revealed that when tDCS is applied to the M1, anodal tDCS seems to locally reduce gammaAminobutyric acid (GABA), while cathodal tDCS causes reduced glutamatergic neuronal activity with a highly correlated reduction in GABA [31]. In addition, it is also reported that anodal tDCS to the premotor cortex facilitates corticomotor excitability, whereas cathodal tDCS does not affect the excitability [17]. Further investigations are needed to better understand cathodal tDCS effects.

Attenuation of the PRP effects under the influence of anodal tDCS was only observed for the 50-ms condition of the CC task (Figure 3(a)). Even under the 50-ms condition, anodal tDCS failed to shorten the RTs in the SS task when simple fixed responses were required (Figure 3(a)). Under the 50-ms condition of the SS task, the same visual stimuli were presented with the same SOA to trigger the finger responses as in the CC task. Thus, it is unlikely that the lopsided RT shortening in the CC task was attributed to general factors such as changes in attentional blinking to the visual stimuli. In addition, the findings rebuffed the view that the tDCS mainly stimulated the M1 so as to simply facilitate the RT regardless of with or without response selection processes. Instead, a plausible interpretation of this finding would be that anodal tDCS to the PMD specifically attenuated the PRP effects, derived from two consecutive choice responses. Since the human PMD seems to be an important brain locus for response selection (see Introduction), the tDCS might benefit this PMD function.

Finally, even in the CC task requiring response selection, anodal tDCS shortened the RT for the 50-ms condition without affecting that for the 500-ms condition. As described, the processes for R1 and R2 should substantially overlap under the 50-ms condition, and this simultaneously forced processing might overload a non-stimulated PMD and generate a significant delay in RT (= PRP effect). Thus, anodal tDCS might reduce this overload by facilitating neuronal processing in the PMD, thereby attenuating the PRP effects under this condition. 
In contrast, the two reaction processes were temporally dissociated at a substantial level under the 500-ms condition, which shortened RT2 as compared with RT1, with no assistance from tDCS. Thus, under this condition, the R2 process was already facilitated, presumably owing to some preparatory effect $[3,32]$. Therefore, the benefits (= RT shortening) from anodal tDCS were little under this condition.

In conclusion, the PRP effects emerged both in RT1 and in RT2 of the CC and SS tasks when the SOA was smaller, but anodal tDCS attenuated the PRP effects, specifically in the CC task requiring two choice responses. Thus, anodal tDCS to the PMD is capable of facilitating its response selection function, and this benefit may reduce PRP effects, particularly when people perform multiple-choice tasks in temporally close succession. This study raised the possibility that non-invasive neuro-modulation to the brain can boost human ability to multi-task, and demonstrated the usefulness of this technique for better understanding of human cognitivemotor functions and their capacities.

\section{Acknowledgements}

This study was conducted as a pre-project for the Institute of Neural Systems and Information Networks. This study was also supported in part by "The Center of Human-Friendly Robotics Based on Cognitive Neuroscience”, a Global COE (Center of Excellence) Program of the Ministry of Education, Culture, Sports, Science and Technology, Japan.

\section{REFERENCES}

[1] R. de Jong, "Multiple Bottlenecks in Overlapping Task Performance,” Journal of Experimental Psychology: Human Perception and Performance, Vol. 19, No. 5, 1993, pp. 965-980. doi:10.1037/0096-1523.19.5.965

[2] H. Pashler, "Dual-Task Interference in Simple Tasks: Data and Theory," Psychological Bulletin, Vol. 116, No. 2, 1994, pp. 220-244. doi:10.1037/0033-2909.116.2.220

[3] M. D. Byrne and J. R. Anderson, "Serial Modules in Parallel: The Psychological Refractory Period and Perfect Time-Sharing,” Psychological Review, Vol. 108, No. 4, 2001, pp. 847-869. doi:10.1037/0033-295X.108.4.847

[4] R. Marois and J. Ivanoff, "Capacity Limits of Information Processing in the Brain," Trends in Cognitive Sciences, Vol. 9, No. 6, 2005, pp. 296-305. doi:10.1016/j.tics.2005.04.010

[5] C. Spence, "Cognitive Neuroscience: Searching for the Bottleneck in the Brain," Current Biology, Vol. 18, No. 20, 2008, pp. 965-968. doi:10.1016/j.cub.2008.08.039

[6] M. C. Lien and R. W. Proctor, "Stimulus-Response Compatibility and Psychological Refractory Period Effects: Implications for Response Selection," Psychonomic Bulletin \& Review, Vol. 9, No. 2, 2002, pp. 212-

\section{8. doi:10.3758/BF03196277}

[7] Y. Jiang, R. Saxe and N. Kanwisher, "Functional Magnetic Resonance Imaging Provides New Constraints on Theories of the Psychological Refractory Period," Psychological Science, Vol. 15, No. 6, 2004, pp. 390-396. doi:10.1111/j.0956-7976.2004.00690.x

[8] T. C. Rickard and D. Bajic, "Memory Retrieval Given Two Independent Cues: Cue Selection or Parallel Access?” Cognitive Psychology, Vol. 48, No. 3, 2004, pp. 243-294. doi:10.1016/j.cogpsych.2003.07.002

[9] R. Marois, J. M. Larson, M. M. Chun and D. Shima, "Response-Specific Sources of Dual-Task Interference in $\mathrm{Hu}$ man Pre-Motor Cortex," Psychological Research, Vol. 70, No. 6, 2006, pp. 436-447. doi:10.1007/s00426-005-0022-6

[10] S. P. Wise and E. A. Murray, "Arbitrary Associations between Antecedents and Actions," Trends in Neurosciences, Vol. 23, No. 6, 2000, pp. 271-276. doi:10.1016/S0166-2236(00)01570-8

[11] E. Koechlin, C. Ody and F. Kouneiher, "The Architecture of Cognitive Control in the Human Prefrontal Cortex," Science, Vol. 302, No. 5648, 2003, pp. 1181-1185. doi:10.1126/science.1088545

[12] E. Koechlin and C. Summerfield, "An Information Theoretical Approach to Prefrontal Executive Function,” Trends in Cognitive Sciences, Vol. 11, No. 6, 2007, pp. 229-235. doi:10.1016/j.tics.2007.04.005

[13] J. O’Shea, C. Sebastian, E. D. Boorman, H. JohansenBerg and M. F. Rushworth, "Functional Specificity of Human Premotor-Motor Cortical Interactions during Action Selection,” European Journal of Neuroscience, Vol. 26, No. 7, 2007, pp. 2085-2095. doi:10.1111/j.1460-9568.2007.05795.x

[14] M. A. Nitsche and W. Paulus, "Excitability Changes Induced in the Human Motor Cortex by Weak Transcranial Direct Current Stimulation,” The Journal of Physiology, Vol. 527, No. 3, 2000, pp. 633-639. doi:10.1111/j.1469-7793.2000.t01-1-00633.x

[15] A. Antal, T. Z. Kincses, M. A. Nitsche, O. Bartfai and W. Paulus, "Excitability Changes Induced in the Human Primary Visual Cortex by Transcranial Direct Current Stimulation: Direct Electrophysiological Evidence,” Investigative Ophthalmology \& Visual Science, Vol. 45, No. 2, 2004, pp. 702-707. doi:10.1167/iovs.03-0688

[16] F. C. Hummel and L. G. Cohen, "Non-Invasive Brain Stimulation: A New Strategy to Improve Neurorehabilitation after Stroke?” Lancet Neurology, Vol. 5, No. 8, 2006, pp. 708-712. doi:10.1016/S1474-4422(06)70525-7

[17] K. Boros, C. Poreisz, A. Munchau, W. Paulus and M. A. Nitsche, "Premotor Transcranial Direct Current Stimulation (tDCS) Affects Primary Motor Excitability in Humans,” European Journal of Neuroscience, Vol. 27, No. 5, 2008, pp. 1292-1300. doi:10.1111/j.1460-9568.2008.06090.x

[18] N. Bolognini, A. Rossetti, C. Casati, F. Mancini and G. Vallar, "Neuromodulation of Multisensory Perception: A tDCS Study of the Sound-Induced Flash Illusion," Neuropsychologia, Vol. 49, No. 2, 2011, pp. 231-237. 
doi:10.1016/j.neuropsychologia.2010.11.015

[19] R. C. Oldfield, "The Assessment and Analysis of Handedness: The Edinburgh Inventory," Neuropsychologia, Vol. 9, No. 1, 1971, pp. 97-113. doi:10.1016/0028-3932(71)90067-4

[20] S. Bestmann, O. Swayne, F. Blankenburg, C. C. Ruff, P. Haggard, et al., "Dorsal Premotor Cortex Exerts StateDependent Causal Influences on Activity in Contralateral Primary Motor and Dorsal Premotor Cortex," Cerebral Cortex, Vol. 18, No. 6, 2008, pp. 1281-1291. doi:10.1093/cercor/bhm159

[21] J. Kroeger, T. Bäumer, M. Jonas, J. C. Rothwell, H. R. Siebner, et al., "Charting the Excitability of Premotor to Motor Connections while Withholding or Initiating a Selected Movement," European Journal of Neuroscience, Vol. 32, No. 10, 2010, pp. 1771-1779. doi:10.1111/j.1460-9568.2010.07442.x

[22] B. W. Vines, N. M. Schnider and G. Schlaug, “Testing for Causality with Transcranial Direct Current Stimulation: Pitch Memory and the Left Supramarginal Gyrus,” Neuroreport, Vol. 17, No. 10, 2006, pp. 1047-1050. doi:10.1097/01.wnr.0000223396.05070.a2

[23] F. Hummel, P. Celnik, P. Giraux, A. Floel, W. H. Wu, et al., "Effects of Non-Invasive Cortical Stimulation on Skilled Motor Function in Chronic Stroke,” Brain, Vol. 128, No. 3, 2005, pp. 490-499. doi:10.1093/brain/awh369

[24] E. K. Kang and N. J. Paik, "Effect of a tDCS Electrode Montage on Implicit Motor Sequence Learning in Healthy Subjects," Experimental \& Translational Stroke Medicine, Vol. 3, No. 1, 2011, p. 4.

[25] J. Reis, H. M. Schambra, L. G. Cohen, E. R. Buch, B. Fritsch, et al., "Noninvasive Cortical Stimulation Enhances Motor Skill Acquisition over Multiple Days through an Effect on Consolidation," Proceedings of the National Academy of Sciences of the United States of America, Vol. 106, No. 5, 2009, pp. 1590-1595. doi:10.1073/pnas.0805413106
[26] G. Corallo, J. Sackur, S. Dehaene and M. Sigman, "Limits on Introspection: Distorted Subjective Time during the Dual-Task Bottleneck,” Psychological Science, Vol. 19, No. 11, 2008, pp. 1110-1117. doi:10.1111/j.1467-9280.2008.02211.x

[27] M. Sigman and S. Dehaene, "Brain Mechanisms of Serial and Parallel Processing during Dual-Task Performance," Journal of Neuroscience, Vol. 28, No. 30, 2008, pp. 75857598. doi:10.1523/JNEUROSCI.0948-08.2008

[28] A. Antal, M. A. Nitsche, T. Z. Kincses, W. Kruse, K. P. Hoffmann, et al., "Facilitation of Visuo-Motor Learning by Transcranial Direct Current Stimulation of the Motor and Extrastriate Visual Areas in Humans," European Journal of Neuroscience, Vol. 19, No. 10, 2004, pp. 28882892. doi:10.1111/j.1460-9568.2004.03367.x

[29] T. Z. Kincses, A. Antal, M. A. Nitsche, O. Bartfai and W. Paulus, "Facilitation of Probabilistic Classification Learning by Transcranial Direct Current Stimulation of the Prefrontal Cortex in the Human,” Neuropsychologia, Vol. 42, No. 1, 2004, pp. 113-117. doi:10.1016/S0028-3932(03)00124-6

[30] N. Lang, H. R. Siebner, Z. Chadaide, K. Boros, M. A. Nitsche, et al., "Bidirectional Modulation of Primary Visual Cortex Excitability: A Combined tDCS and rTMS Study," Investigative Ophthalmology \& Visual Science, Vol. 48, No. 12, 2007, pp. 5782-5787. doi:10.1167/iovs.07-0706

[31] C. J. Stagg, J. G. Best, M. C. Stephenson, J. O’Shea, M. Wylezinska, et al., "Polarity-Sensitive Modulation of Cortical Neurotransmitters by Transcranial Stimulation,” Journal of Neuroscience, Vol. 29, No. 16, 2009, pp. 5202-5206. doi:10.1523/JNEUROSCI.4432-08.2009

[32] A. Howes, R. L. Lewis and A. Vera, "Rational Adaptation under Task and Processing Constraints: Implications for Testing Theories of Cognition and Action,” Psychological Review, Vol. 116, No. 4, 2009, pp. 717-751. doi:10.1037/a0017187 